\title{
A novel approach to enhance the accuracy of vibration control of Frames
}

\author{
Iraj Toloue ${ }^{1 *}$, Mohd Shahir Liew ${ }^{1}$, I.S.H Harahap ${ }^{1}$, and H.E. Lee ${ }^{1}$ \\ ${ }^{1}$ Civil and Environmental Engineering Department, Universiti Teknologi PETRONAS, Bandar Seri \\ Iskandar, 32610 Seri Iskandar Perak, Malaysia
}

\begin{abstract}
All structures built within known seismically active regions are typically designed to endure earthquake forces. Despite advances in earthquake resistant structures, it can be inferred from hindsight that no structure is entirely immune to damage from earthquakes. Active vibration control systems, unlike the traditional methods which enlarge beams and columns, are highly effective countermeasures to reduce the effects of earthquake loading on a structure. It requires fast computation of nonlinear structural analysis in near time and has historically demanded advanced programming hosted on powerful computers. This research aims to develop a new approach for active vibration control of frames, which is applicable over both elastic and plastic material behavior. In this study, the Force Analogy Method (FAM), which is based on Hook's Law is further extended using the Timoshenko element which considers shear deformations to increase the reliability and accuracy of the controller. The proposed algorithm is applied to a $2 \mathrm{D}$ portal frame equipped with linear actuator, which is designed based on full state Linear Quadratic Regulator (LQR). For comparison purposes, the portal frame is analysed by both the Euler Bernoulli and Timoshenko element respectively. The results clearly demonstrate the superiority of the Timoshenko element over Euler Bernoulli for application in nonlinear analysis.
\end{abstract}

\section{Introduction}

Smart structures are those structures equipped with sensors and computers, which enable them to sense the environmental dynamic loadings such as earthquakes. Additional devices such as actuators along with installed sensors help structures to modify their behavior in real time and bear more loads [1]. This method is typically in relative contrast to the traditional body of knowledge of structural engineering [2]. It is worth nothing that in the past thirty years, the interest on integrating actuators with structures to provide controlled forces to compensate applied external loads and minimize structural responses have increased [3]. All of these structures required a suitable algorithm to determine the magnitude of the required forces to reduce the structural response in real time.

\footnotetext{
* Corresponding author: toloue.iraj@gmail.com
} 
Active tendon systems (ATS), are composed of pre-stressed cables, which connect the floors of the structures in a crosswise manner with an actuator. The Actuator is connected to the pre-stressed cables, and its function is primarily to vary the level of the tension in the cables (see Figure.3). As a result the response of the structure is minimized in this system [4].

Rodllar et.al, by employing an ATS on a cable steady bridge attempted to show the system's efficiency. The linear controller was derived based on the algebraic Lyapunov equation. A finite element numerical example of a $142.5 \mathrm{~m}$ long pedestrian cable steady bridge, subjected to the 1952 Taft earthquake, showcased a significant reduction in the bridge response [5].

Alavinasab et,al. proposed a new approach to optimize the linear quadratic regulator (LQR) controller employed in an active tendon control system to achieve minimum top node displacement. Their method was derived similar to the LQR method, but based on the energy technique. Numerical simulations comprising, a two dimensional and a three-story frame with two ATS, subjected to variety of earthquakes were performed. The study used lumped mass system to derive the gain matrices and retrieved with notable displacement attenuation [6].

Atig et,al. showed the possibility of controlling the instability of tensegrity systems even during nonlinear analysis by ATS. To obtain the nonlinear analysis the iterative Newton Raphson method is used [7]. Therefore, it can be deduced that nonlinear analysis is one of the key methods in vibration analysis, which involves complex algorithms from being computationally expensive. Consequently, advanced and powerful computers are required for such analysis. To circumvent this setback there are notably new alternative methods, which can replace the traditional methods to run faster analyses with sufficient accuracy. This paper highlight the use of Force Analogy Method (FAM) introduced by Lin in 1968 for inelastic behavior in continuum mechanics[8]. FAM was later further developed by Wong and Yang for structural analysis purpose which instead of tracking the changes in stiffness, used the displacement variations to represent the inelastic behavior of the system [9].

To demonstrate the accuracy of the FAM, Wong and Yang, applied the method on a two- dimensional six story hospital building subjected to 1994 Northridge earthquake. Uniform damping matrix, lumped mass system, and the general assumption of the FAM applied in the simulation. The study's results were proven to be sufficient to compute the true plastic hinges [10]. Wang and Wong, introduced the FAM for stochastic dynamic analysis of structures at the inelastic zone. They used the structural responses to generate the variance covariance function to enable the method to be in good agreement with earthquake analysis [11]. Qu and $\mathrm{Li}$, applied the FAM to incorporate weighting coefficients in developing a new location index model. This approach intends to reveals optimized location of dampers. The performance index was based on the plastic energy of each member as computed from the derived FAM. The accuracy of the FAM was shown in retrieving hysteric energy for a structural system equipped with passive control devices [12].

Wong and Li also proposed a few methods to demonstrate the implementation of FAM for frames equipped with passive, semi active, and active controllers. All of the models were verified for their representation accuracies of the systems [13]. The structural responses such as P- $\Delta$ effect, and calculated displacement by FAM, are typically computed based on the Euler Bernoulli beam element [14]-[16]. The 
Euler Bernoulli element is derived based on flexural considerations only while rotary inertia and shear effects are both neglected [17]. The Timoshenko element, on the other hand includes the aforementioned neglected assumptions [18]. Many researches including Clough believed that the Euler Bernoulli element, is sufficient for accuracy requirements of general civil engineering analysis [19]. Dixit, who performed structural damage detection, which required accurate analysis compared results based on Timoshenko element of a damaged single beam with that of Euler Bernoulli. The results indicate differences in computed natural frequencies of the elastic structure [20]. Mei performed vibration analysis on a multi-story frame based on the Timoshenko element. The natural frequency responses of the analyzed frame was studied for "L", and " $T$ " shape joints. The results are in good agreement with classical elements [21].

In this paper, the computed difference from using the Euler Bernoulli, and Timoshenko element on nonlinear active vibration controlled frames analyzed by FAM is given full treatment. An LQR controller and state space method coded in MATLAB is developed to perform the nonlinear analyses. An iterative loop is built into the algorithm to uncover the phase change effect, when the wrong element selected.

\section{Methodology}

The equation of motion for a structure equipped with ATC, is shown in Equation 1.

$$
M \ddot{x}+C \dot{x}+K x=F_{e}+F_{C}
$$

Where $\mathrm{M}, \mathrm{C}, \mathrm{K}, \mathrm{F}_{\mathrm{e}}$, and $\mathrm{F}_{\mathrm{c}}$ are mass, damping, stiffness, external force, and control force matrices. The state space form of Equation 1 is written as Equation 2.

$$
Z_{t+\Delta t}(t)=F_{d} Z_{t}(t)+H_{d} a(t)+G_{d} X^{\prime \prime}+G_{c} f_{c k}
$$

Where $Z_{t+\Delta t}(t)$ is the state matrix at time " $\mathrm{t}$ ", $\mathrm{a}(\mathrm{t})$ is the base acceleration, " $\mathrm{D}$ " is the base acceleration and the ATC distribution control matrix, $X$ " is the inelastic displacement, and $f_{c k}$ is the ATC force matrix which are numerically denoted in the following: $F_{d}=$ $e^{A \Delta t}, A=\left[\begin{array}{cc}0 & I \\ -K / M & -C / M\end{array}\right], G=\left[\begin{array}{c}0 \\ K / M\end{array}\right], G_{c}=F_{d} D \Delta t, G_{d}=F_{d} G \Delta t, H_{d}=F_{d} H \Delta t$.

The fundamental concept of the FAM, is shown in Fig. 1. The initial stiffness, post yielding stiffness, yielding force, yielding displacement, total force, and total displacement denoted by $\mathrm{K}_{\mathrm{i}}, \mathrm{K}_{\mathrm{py}}, \mathrm{F}_{\mathrm{y}}, \mathrm{X}_{\mathrm{y}}, \mathrm{Ft}, \mathrm{X}$, respectively.

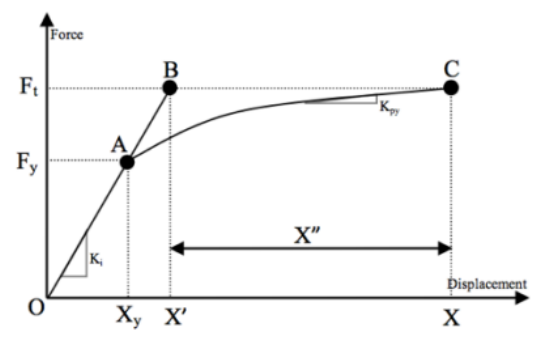

Fig. 1. Force-Displacement relationship. 
By extending the OA line from yielding force to the total force (point B), the elastic displacement is determined. The difference between the total displacement and the elastic displacement as shown in Equation 3 , defines the inelastic displacement.

$$
X^{\prime \prime}=X-X^{\prime}
$$
4.

The same concept for Equation 3, is applicable for moment which leads to the Equation

$$
m^{\prime \prime}=m-m^{\prime}
$$

Equations 3 and 4 show that it is possible to ascertain the nonlinear behaviour of a system by solely relying on the initial stiffness. This is extremely useful as it enables avoidance of additional computational effort to repetitively calculate the post yielding stiffness matrix.

The plastic rotation at individual members of a structure can be replaced by a set of imaginary moments and forces, which are shown in Equations 5 and Error! Reference source not found., respectively.

$$
\begin{gathered}
F_{\text {RForce }}=-K^{\prime} \theta^{\prime \prime} \\
M_{\text {RForce }}=-K^{\prime \prime} \theta^{\prime \prime}
\end{gathered}
$$

The combination of the Equations 3, 5 and the stiffness method results in a fully linear method to ascertain the inelastic displacement, which is shown in Equation 7.

$$
X^{\prime \prime}=K^{-1} K^{\prime} \theta^{\prime \prime}
$$

In a same way the inelastic moment can be determined as shown in Equation 8.

$$
m^{\prime \prime}=-\left(K^{\prime \prime}-K^{\prime T} K^{-1} K^{\prime}\right) \theta^{\prime \prime}
$$

Finally by combining the Equations 4, Error! Reference source not found., 7, and 8 a relation to compute the moment at the predefined plastic hinge locations (PHL), is shown in Equation 9.

$$
m=K^{\prime T} x-K^{\prime \prime} \theta^{\prime \prime}
$$

The plastic rotation vector $\theta^{\prime \prime}$ is zero at the initially and if the moment computed via in Equation 9, exceeds the material yielding limit, the rotation vector will be calculated based on Equation 10.

$$
\theta^{\prime \prime}=K^{\prime \prime-1}\left(K^{\prime T} x-m\right)
$$

Where based on the Timoshenko element the stiffness matrices are illustrated in Equations 11 and 12.

$$
K=\left[\begin{array}{ccccc}
\frac{12 E I}{L^{3}(1+\varphi)} & \frac{6 E I}{L^{2}(1+\varphi)} & \frac{-12 E I}{L^{3}(1+\varphi)} & \frac{6 E I}{L^{2}(1+\varphi)} \\
\frac{6 E I}{L^{2}(1+\varphi)} & \frac{(4+\varphi) E I}{L(1+\varphi)} & \frac{6 E I}{L^{2}(1+\varphi)} & \frac{(2+\varphi) E I}{L(1+\varphi)} \\
\frac{-12 E I}{L^{3}(1+\varphi)} & \frac{6 E I}{L^{2}(1+\varphi)} & \frac{12 E I}{L^{3}(1+\varphi)} & \frac{6 E I}{L^{2}(1+\varphi)} \\
\frac{6 E I}{L^{2}(1+\varphi)} & \frac{(2+\varphi) E I}{L(1+\varphi)} & \frac{6 E I}{L^{2}(1+\varphi)} & \frac{(4+\varphi) E I}{L(1+\varphi)}
\end{array}\right]
$$




$$
K^{\prime}=\left[\begin{array}{ll}
\frac{6 E I}{L^{2}(1+\varphi)} & \frac{6 E I}{L^{2}(1+\varphi)} \\
\frac{(4+\varphi) E I}{L(1+\varphi)} & \frac{(2+\varphi) E I}{L(1+\varphi)} \\
\frac{-6 E I}{L^{2}(1+\varphi)} & \frac{-6 E I}{L^{2}(1+\varphi)} \\
\frac{(2+\varphi) E I}{L(1+\varphi)} & \frac{(4+\varphi) E I}{L(1+\varphi)}
\end{array}\right], K^{\prime \prime}=\left[\begin{array}{ll}
\frac{(4+\varphi) E I}{L(1+\varphi)} & \frac{(2+\varphi) E I}{L(1+\varphi)} \\
\frac{(2+\varphi) E I}{L(1+\varphi)} & \frac{(4+\varphi) E I}{L(1+\varphi)}
\end{array}\right], \varphi=\frac{12 E I}{G \frac{A}{\kappa} L^{2}}
$$

In the stiffness matrix, modulus of elasticity and moment of inertia are shown by "E", and "I". Length, cross section area, shear modulus, and Timoshenko shear coefficient are respectively represented by " $L$ ", " $A$ ", "G", and " $\kappa$ ".

The Variable $f_{c k}$ in Equation 2 is the required actuator force to control the system's vibration based on the LQR system. The LQR is modelled by defining a cost function to minimize the system's response, as shown in Equation 13.

$$
J=\frac{1}{2} \int_{0}^{\infty} Z^{T} Q Z+f_{c}^{T} R f_{c}
$$

Control and state variables are represented by " $f_{c}$ ", and " $Z$ ". The weighting matrices "Q", and "R" are usually obtained through an iterative trial and error process. In this study the weighting matrices are chosen based on the previous literatures. The minimization of cost function, and discrete algebraic RICCATI equation (DARE), the " $f_{c k}$ " is obtained by Equation 14.

$$
f_{c k}=\frac{G_{c}{ }^{T} \times P_{B} \times f_{d} \times Z_{0}}{G_{c}{ }^{T} \times P_{B} \times G_{c}+R}
$$

" $P_{B}$ " is the result of the DARE. The process algorithm of this study is shown in Fig. 2.

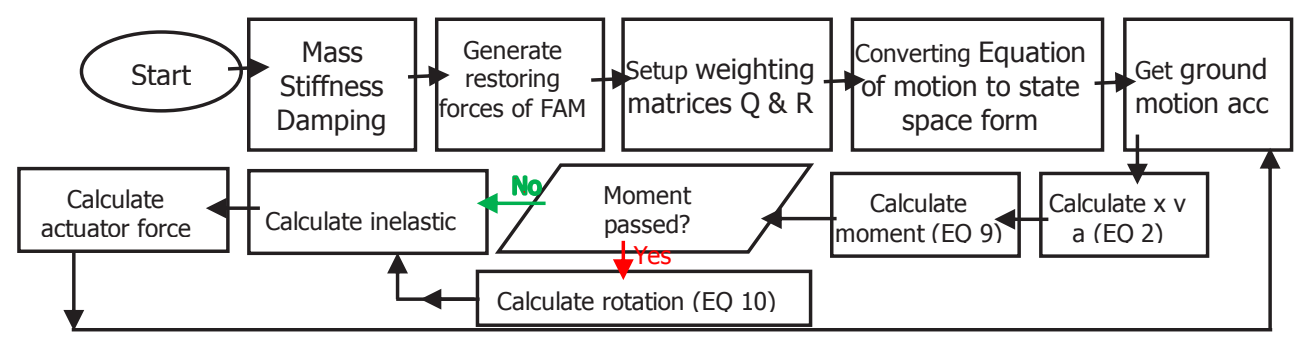

Fig. 2. Flowchart for proposed algorithm.

\section{Applications}

The portal frame and the node numbering are shown in Fig. 3. This frame model is similar to previous studies for convenience of benchmarking [22-23]. As illustrated, the actuator is connected to the Nodes number 2 and 4 . There are two PHL defined on both end of each member. The actuator movement to the right side considered as positive. The section details for both beam and columns are a cubic hallow section and it is shown in Fig. 4. 


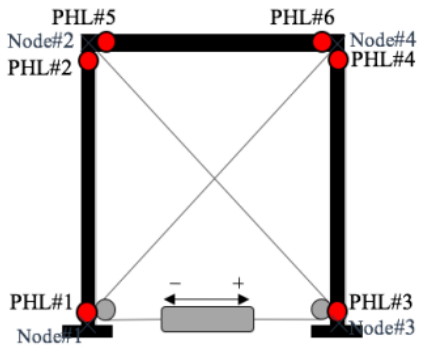

Fig. 3. Proposed model.

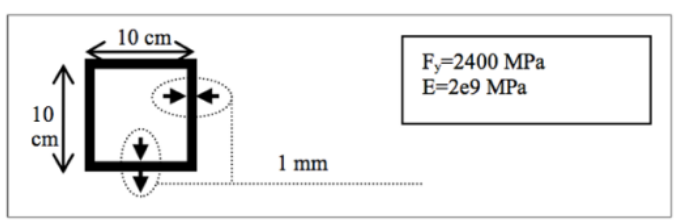

Fig. 4. Section details.

Finally the frame model in Fig. 3 is subjected to ground motion from the 1940 El-Centro earthquake which is shown in Fig. 5.

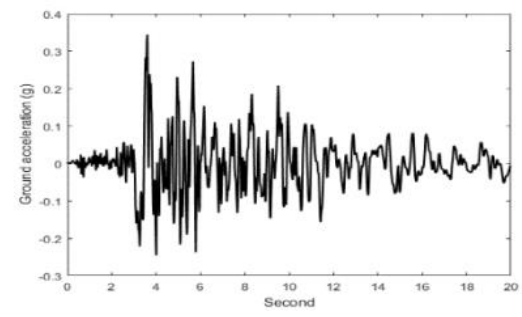

Fig. 5. Ground acceleration.

\section{Results and discussions}

The proposed frame is analyzed and controlled by both Timoshenko, and Euler Bernoulli elements. The non-linear rotations of the frame at all of the defined PHLS are shown in Figure 6 . Each of the graphs, separately show the two end plastic hinges results for one element (two columns and one beam).
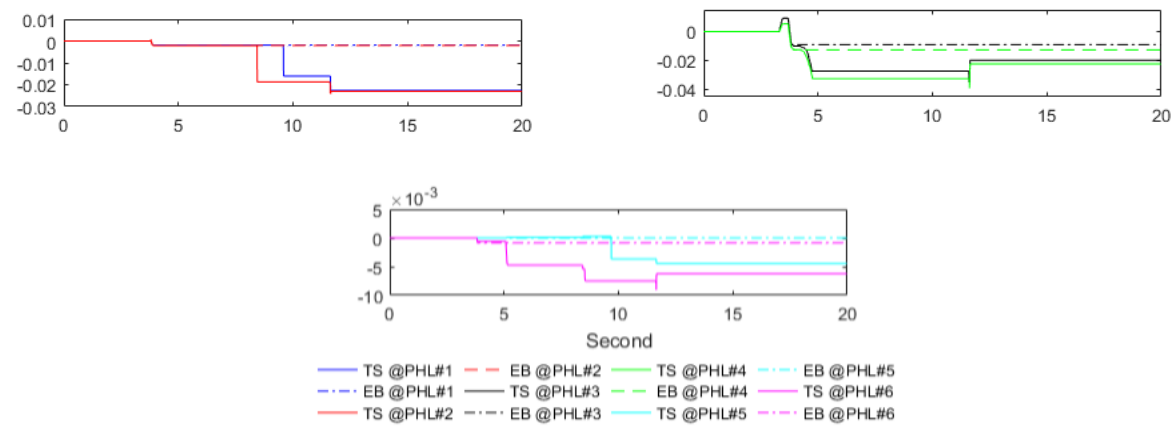

Fig. 6. PHL at all 6 nodes with different element type.

It is shown in the previous study that the results of the analysis based on the Timoshenko (TS), and the Euler Bernoulli (EB) elements are not similar. Moreover, the differences between these two elements are not significant in terms of magnitude. The maximum differences between EB and TS element are shown in Table 1. The differences between TS, and EB, in respect to EB element, are shown in last column of Table 1, and Figure 7. 
Table 1. Nonlinear rotations in respect to EB element.

\begin{tabular}{|c|c|c|c|}
\hline PHL & EB (rad) & TS (rad) & Difference (\%) \\
\hline$\# 1$ & -0.0227 & -0.0019 & 91.746 \\
\hline$\# 2$ & -0.0353 & -0.0090 & 74.5410 \\
\hline$\# 3$ & -0.0242 & -0.0020 & 91.5803 \\
\hline$\# 4$ & -0.0391 & -0.0126 & 67.8186 \\
\hline$\# 5$ & -0.0045 & 0 & 100 \\
\hline$\# 6$ & -0.0091 & -0.008 & 90.6776 \\
\hline
\end{tabular}

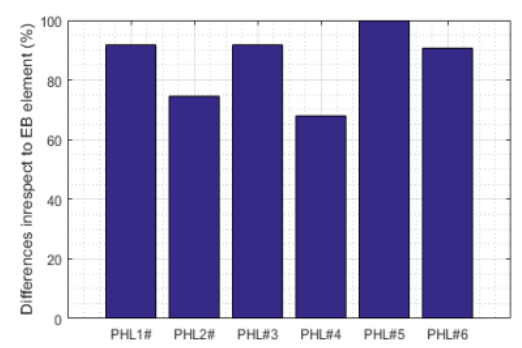

Fig. 7. Differences between EB and TS element.

It was expected due to the symmetrical frame shape, the results on $\mathrm{PHL} \# 1,2$, and 5, be almost the same as the PHL\#3, 4, and 6, respectively. However, Fig. 7, does not substantiate this hypothesis, and proving that all of the individual PHLs are required to be calculated separately. On the other hand, scrutinizing to the applied control force, as it is shown in Fig. 8, the changed phase between the Euler Bernoulli and Timoshenko element can be clearly seen. A close look at the control force between 7.9 to 8.1 seconds show that the phases of the control force based on these two elements are in the opposite direction of each other. In other words, the actuator based on the EB element moved towards the left with $-1 \mathrm{kN}$, while the actuator based on TS moved to the right with $+1 \mathrm{kN}$. For the time frame between 9.75 to 11.0 seconds, the EB case actuator saturated at the left side with $-1 \mathrm{kN}$, while TS case actuator force is fluctuates between the maximum levels of both left and right directions.
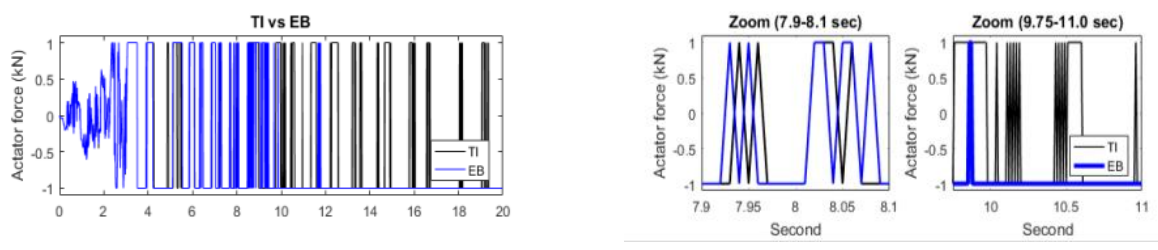

Fig. 8. Control force with different element type.

The displacement results of the system in $X$ and $Z$ directions are reported in Fig. 9 and Fig. 10, respectively. Although the analysis results represent a shift in the displacement, but there are only minor differences between the amplitude of vibration of these two elements. According to Clough this small amount of difference is negligible in general practice of structural engineering [24]. 

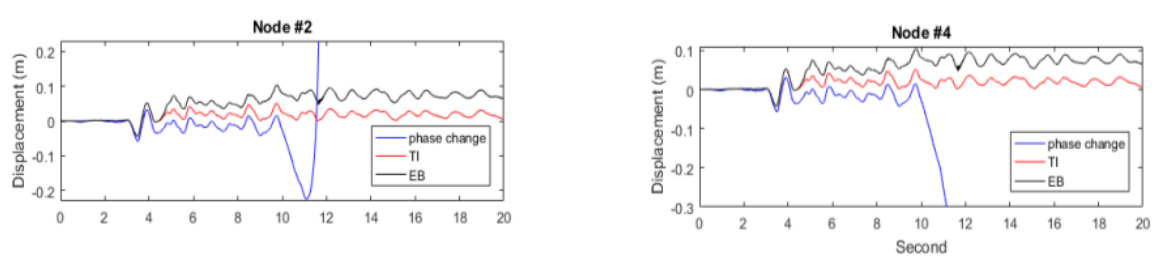

Fig. 9. Displacement in X-direction with different element type.

The frame response is computed again with TS element in lieu of EB for more accuracy. The actuator, as a common practice for FAM, is controlled by the EB element rather than TS. The results indicated structural collapse. The failure occurred when the phase change of the actuator is taken into account. Figure 8 showcases the start of collapse which can be deduced to be at the time 7.9 seconds.

It is much clearer to see the differences between these two elements in Fig. 10. The highlighted periods at Fig. 8, at the time $7.9 \mathrm{sec}$, and $9.75 \mathrm{sec}$, are obvious in Figure 10. Lastly, it can be additionally remarked for this study that the recorded response in the $Y$ direction is too small to pose any threat to the structure's health.
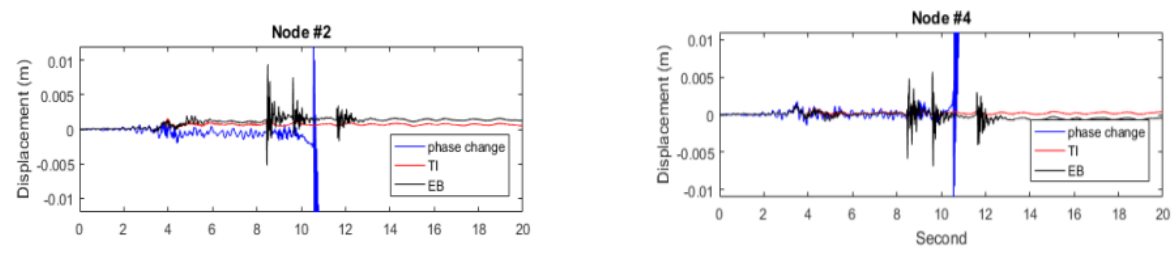

Fig. 10. Displacement in Z-direction with different element type.

As the Timoshenko element, unlike the Euler Bernoulli, consider the shear deformations, the accuracy of the FAM on calculation of rotations at PHL increased. Moreover, the small changes in displacement amplitude return a huge difference in the phase of the actuator force. This phase change continued to some extend that the state space results in prediction of the structural response in time " $k+1$ " to return error and trigger structural collapse.

\section{Conclusions}

In this study, a numerical 2D portal frame is equipped with an actuator is subjected to the 1940 El-Centro earthquake. The nonlinear analysis on this frame was performed by FAM, which is based on the EB element and TS elements respectively. Minor differences in structural response between the use of EB and TS elements were detected when the actuator phase changes are neglected. As the TS consider the neglected shear deformations of the EB element, such small changes were captured by the model which resulted in the phase of the actuator for EB and TS element to be acting in the opposite direction of each other. When the phase change of the actuator was taken into consideration the structure was noted to have collapsed.

\section{References}


1. H. Adeli, X. Jiang, Intelligent infrastructure: neural networks, wavelets, and chaos theory for intelligent transportation systems and smart structures. (CRC Press, 2009)

2. L.-Y. Lu, G.-L. Lin, C.-H. Lin, Comput. Civ. Infrastruct. Eng. 24(1), 41-61, (2009)

3. H. Adeli, A. Saleh, Control, Optimization, and Smart Structures-HighPerformance Bridges and Buildings of the Future, (John Wiley and Sons, 1999)

4. T. E. Saaed, G. Nikolakopoulos, J.-E. Jonasson, H. Hedlund, J. Vib. Control 21(5), 919-937, (2013)

5. J. Rodellar, V. Manosa, C. Monroy, J. Struct. Control, (2002)

6. A. Alavinasab, H. Moharrami, A. Khajepour, Comput. Civ. and Infrast. Eng. 21(8), 605-611 (2006)

7. M. Atig, M. H. El Ouni, N. Ben Kahla, Eur. J. Environ. Civ. Eng. 8189 (March), $1-18,(2017)$

8. T. H. Lin, Theory of Inelastic Structures. (New York, NY, USA: John Wiley \& Sons, 1968)

9. K. K. F. Wong, R. Yang, J. Eng. Mech., 125(10), 1190-1199 (1999)

10. K. K. F. Wong, R. Yang, J. Eng. Mech., 128, 308-317 (2002)

11. Z. Wang, K. K. F. Wong, J. Eng. Mech., 133(4), 414-421, (2007)

12. J. Qu, H. Li, Math. Probl. Eng., 2013, 10, (2013)

13. Y. Chuan-guo, L. Chun-guang, X. Chun-Xu, J. Water Resour. Archit. Eng., 3(3), 72-75, (2014)

14. Y. Zhang, H.N. Li, G. Li, Int. J. Struct. Stab. Dyn., 16(5), 1550012, (2016)

15. G. Li, H.N. Li, Y. Zhang, Struct. Des. Tall Spec. Build., 24, 59-72 (2015)

16. G. Li, L. A. Fahnestock, H.N. Li, J. Struct. Eng., 139(4), 500, (2012)

17. T. M. Wang, T. A. Kinsman, J. Sound Vib., 14(2), 215-227, (1971)

18. R. J. Astley, Finite elements in solids and structures. An introduction. (Chapman \& Hall (Springer), 1992)

19. R. W. Clough, Bull. Seismol. Soc. Am., 45 (4), 289-301, (1955)

20. A. Dixit, J. Sound Vib., 333(18), 4341-4353, (2014)

21. C. Mei, J. Dyn. Syst. Meas. Control, 136(5), 51004, (2014)

22. K. K. F. Wong, G. Li, Theory of Nonlinear Structural Analysis: The Force Analogy Method for Earthquake Engineering, (John Wiley and Sons, 2014).

23. G. C. Hart, K. Wong, Structural Dynamics for Structural Engineers. (New York, John Wiley and Sons, 2000)

24. R. W. Clough, J. Penzien, Dynamics of structures, Third. (California, 2003) 